\title{
UHF IGZO Schottky diode
}

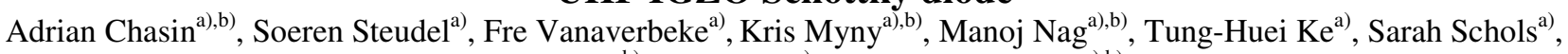
Georges Gielen ${ }^{\mathrm{b}}$, Jan Genoe ${ }^{\mathrm{a})}$, and Paul Heremans ${ }^{\mathrm{a}, \mathrm{b})}$

a) imec, Kapeldreef 75, 3001, Leuven, Belgium; b) ESAT, KU Leuven, Kasteelpark Arenberg 10, 3001, Leuven, Belgium

High-performance Schottky diodes based on amorphous IGZO (Indium-Gallium-Zinc Oxide) semiconductors were fabricated and fully characterized. S-parameter measurements and subsequent analysis prove that these diodes have a cutoff frequency over $900 \mathrm{MHz}$, making these diodes an excellent choice for UHF applications, such as energy-harvesters for passive RFID tags on foil. Moreover, when used in a single stage rectifier, the diodes provide DC voltages higher than $1.2 \mathrm{~V}$ at $900 \mathrm{MHz}$, which is enough supply for circuitry on foil based on current metal-oxides technologies [1,2].

Thin-film circuits fabricated directly on plastic foil [2] are contenders for low-cost 'plastic' electronic tags. Preferably, such tags are passive, i.e. powered by the electromagnetic wave sent by the receiver. To that end, a rectifier is required on the tag. In the past it was shown that organic semiconductor rectifiers can operate at $\mathrm{HF}(13.56 \mathrm{MHz})[3,4]$; to reach UHF $(\sim 900 \mathrm{MHz})$, we here propose rectifiers based on amorphous metal-oxide semiconductors such as IGZO, as they combine a higher intrinsic mobility and air stability while still allowing processing with low thermal budgets compatible with plastic substrates [5].

Pd electrodes were deposited and patterned by photolithography on a glass substrate. A 260nm thick amorphous IGZO layer was subsequently deposited by rf sputtering from a $\mathrm{InGaZnO}_{4}$ ceramic target at room temperature. The oxygen content of the $\left(\mathrm{Ar}+\mathrm{O}_{2}\right)$ gases was maintained at $20 \%$ and the working pressure in the deposition chamber was $2 \times 10^{-2}$ mbar. The IGZO layer was wet etched in a solution of oxalic acid and water. As final step, Ohmic Mo top contacts with different sizes were also formed by a lift-off process, providing vertical diodes with areas from $20 \times 10 \mu \mathrm{m}^{2}$ to $220 \times 200 \mu \mathrm{m}^{2}$ (Figure 1a).

The high $\mathrm{O}_{2}$ pressure is one of the requisites to obtain a Schottky barrier, because it reduces the IGZO subgap surface states and, consequently, the Fermi level pinning. The obtained Schottky behavior is visible in Figure 1b. We achieved a further improvement of the off-current by performing an in-situ $\mathrm{O}_{2}$ plasma treatment on the Pd bottom electrode prior to IGZO deposition (Figure 1c). A final post-annealing in $1 \mathrm{~atm} \mathrm{O}_{2}$ at $200^{\circ} \mathrm{C}$ reduces even further the off-current, while still enabling a high on-current level. The final devices have rectification ratios higher than $10^{8}$ at $\pm 1 \mathrm{~V}$, current densities of up to $100 \mathrm{~A} / \mathrm{cm}^{2}$ at forward bias of $2 \mathrm{~V}$ and reverse breakdown voltages higher than 10V, as shown in Figure 2. State of art organic Schottky diodes, on the other hand, have much smaller rectification ratios at $\pm 1 \mathrm{~V}$ of up to 50 [3] and $10^{4}$ [4]. Figure 3 shows the on and off-current dependency on the diodes' area and the homogeneity of the devices over a $3 \times 3 \mathrm{~cm}^{2}$ sample. Although some spread of the current levels is seen, the rectification ratio is constant around $10^{8}$ for all devices. Our diodes are the first IGZO Schottky diodes in which the charge transport mechanism can be explained via a pure thermionic emission process over the Schottky barrier. We prove this by modeling the measured forward current of the diodes by the thermionic emission model with the parameters shown in Table I over the whole forward bias regime (Figure 4). Temperature dependent IV measurements are shown in Figure 5a with its respective Richardson plot (Figure 5b), confirming the uniformity of the Schottky barrier.

The inset of Figure 6 shows that capacitance of the diode is almost constant at negative bias, because the IGZO film is fully depleted at negative voltages. The obtained $6 \mathrm{pF}$ corresponds to the geometrical capacitance of the semiconductor layer. Therefore, we extracted the relative dielectric constant $\varepsilon_{S}$ of IGZO to be 14.9. Based on this value and the CV measurements shown in Figure 6, it is possible to extract the built-in potential $\mathrm{V}_{\mathrm{bi}}$ and the ionized atoms concentration within the depletion region $\mathrm{N}_{\text {depl }}$. In covalent semiconductors, the depletion region of a Schottky diode reveals the ionized dopant concentration which is fully correlated to the free charge concentration. In amorphous semiconductors, however, due to subgap traps, the depletion region also reveals ionized atoms that do not contribute to the free charge concentration. Therefore, $\mathrm{N}_{\mathrm{depl}}$ corresponds to subgap trap density. The free charge concentration $\mathrm{N}_{\mathrm{e}}$, on the other hand, is extracted from the resistivity of the diodes obtained from IV measurements by assuming a mobility equal to $10 \mathrm{~cm}^{2} /(\mathrm{V} . \mathrm{s})$.

One-port scattering parameter measurements were taken of the diodes. $S_{11}$ of a $120 \times 100 \mu \mathrm{m}^{2}$ area diode is shown in Figure 7 at different bias voltages for frequencies ranging from $10 \mathrm{MHz}$ to $10 \mathrm{GHz}$. The top-left inset shows the small-signal model used to extract the various device components: a parallel variable resistance $\left(\mathrm{R}_{\mathrm{b}}\right)$ that depends on the applied bias and a capacitance $\left(C_{b}\right)$, representative of the diode's barrier, in series with a second resistance $\left(r_{s}\right)$, representing any series resistance due to contacts. A photograph of the device used for RF measurement is shown on the top-right inset.

At sufficiently high frequency the junction capacitance dominates the barrier conductance. Accordingly, a useful "figure-ofmerit" has proved to be the frequency at which $1 / \omega \mathrm{C}_{\mathrm{b}}$ equals $\mathrm{r}_{\mathrm{s}}$, which is called the cutoff frequency. Figure 8 shows the value of $r_{s}$ and $X_{b}$ extracted from $S_{11}$ at $0 \mathrm{~V}$ as a function of the frequency. It is clear that the diode has a RC-cutoff frequency over $900 \mathrm{MHz}$. The inset shows $\mathrm{C}_{\mathrm{b}}$ extracted from $\mathrm{X}_{\mathrm{b}}$ as a function of the frequency: its value is the same as obtained previously from $\mathrm{CV}$ measurements with the diode in negative bias shown in Figure 6.

Finally, the diode was connected in a single stage rectifier using a $100 \mathrm{nF}$ capacitor and $1 \mathrm{M} \Omega$ load resistance. A $50 \Omega$ resistor was used for proper termination of the incoming RF signal (Figure 9a). Figure 9b presents the average DC component of the rectified output signal $\left(\mathrm{V}_{\text {out }}\right.$ ) obtained for an incoming sine wave with amplitudes of $3 \mathrm{~V}$ (i.e. $6 \mathrm{~V}$ peak-to-peak) or $5 \mathrm{~V}$ (i.e. $10 \mathrm{~V}$ peak-to-peak) for different frequencies. For a $1 \mathrm{MHz}$ signal, stable rectified DC outputs of $2.4 \mathrm{~V}$ (Figure $10 \mathrm{~b}$ ) and $4.5 \mathrm{~V}$ are obtained, for $\mathrm{AC}$ input of $3 \mathrm{~V}$ and $5 \mathrm{~V}$, respectively. At $800 \mathrm{MHz}$, the $\mathrm{DC}$ values dropped to $0.37 \mathrm{~V}$ and $1.26 \mathrm{~V}$ as shown in Figure 10d. Comparing to organic diodes, IGZO diodes supplies more voltage at the same frequency due its low turn-on voltage (low $\mathrm{V}_{\mathrm{bi}}$ ) and high rectification ratios.

In summary, we have demonstrated for the first time high performance, low turn-on and ultra-fast IGZO Schottky diodes, with $\mathrm{f}_{\mathrm{RC}}$ over $900 \mathrm{MHz}$ that supplies more than $1 \mathrm{~V}$ DC at UHF regime.

[1] M. Rockele et al., Organic Electronics 12, 1909 (2011). [2] A. K. Tripathi et al., Appl. Phys. Lett. 98, 162102 (2011). [3] S. Steudel et al., IEDM Tech.Dig., (2008). [4] D. Im et al., Adv. Mater., 23, 644 (2011). [5] K. Nomura et al., Nature 432, 488 (2004). 


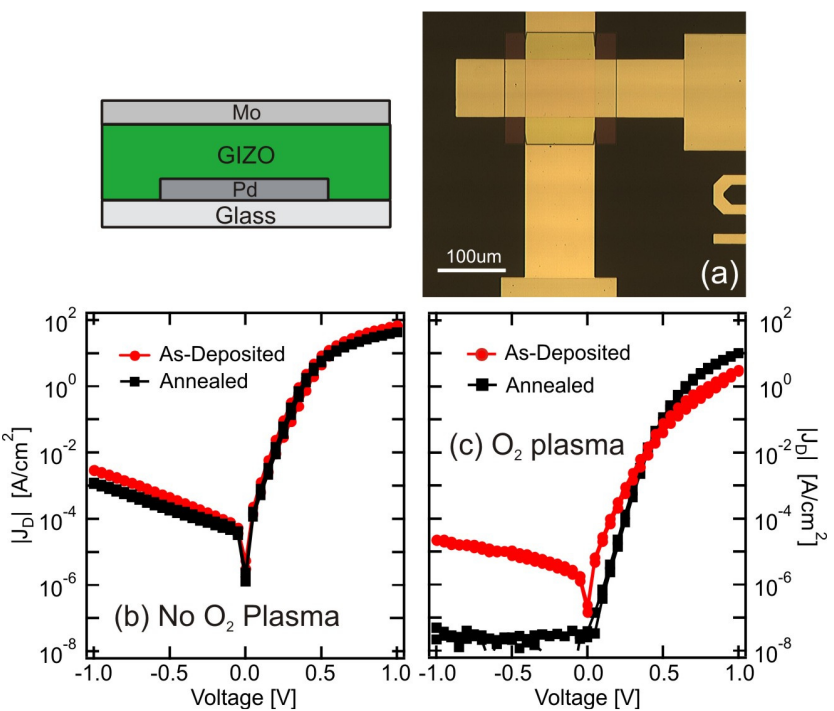

Figure 1: (a) Top-view image and cross-section schematic of the IGZO diodes' topology. The influence of the Pd bottom contact pre-treatment and the annealing step on the diode characteristics are shown on (b) and (c). The diode area is $120 \times 100 \mu \mathrm{m}^{2}$.

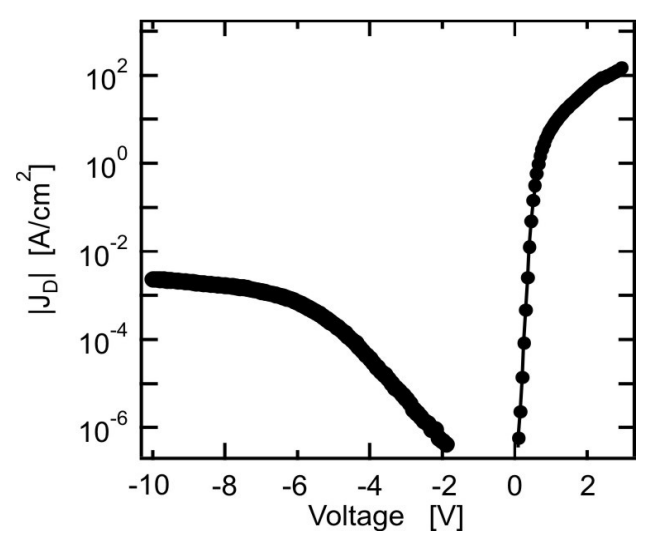

Figure 2: Wide bias curve showing the diode's typical forward and reverse current density at $+2 \mathrm{~V}$ and $-10 \mathrm{~V}$, respectively. The diode area is $120 \times 100 \mu \mathrm{m}^{2}$.

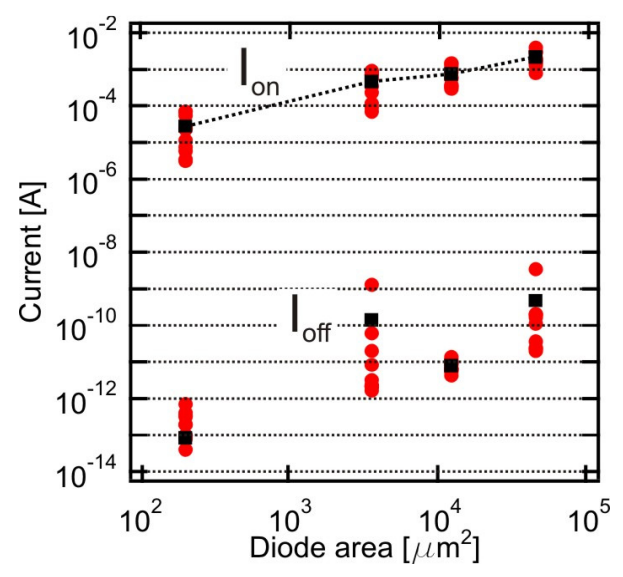

Figure 3: On and off-current for diodes with different sizes at $\mathrm{V}= \pm 1 \mathrm{~V}$ within a sample of $3 \times 3 \mathrm{~cm}^{2}$. There are 10 devices measured for each size and the black squares are the average value.

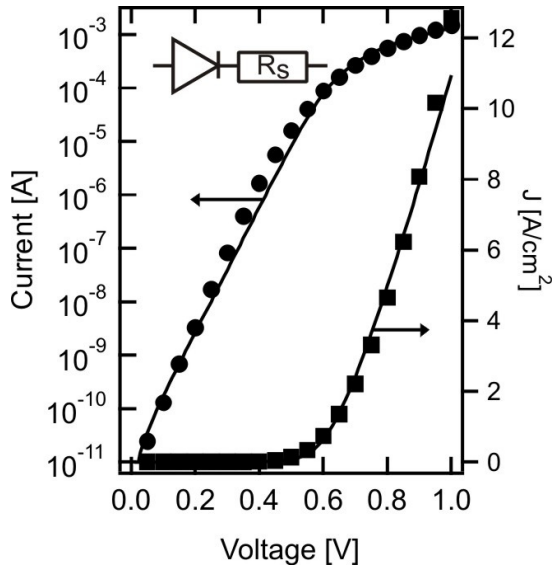

Figure 4: Forward current experimental data (symbols) and fit with a pure thermionic emission model with the parameters from Table I (full lines). The diode area is $120 \times 100 \mu \mathrm{m}^{2}$.

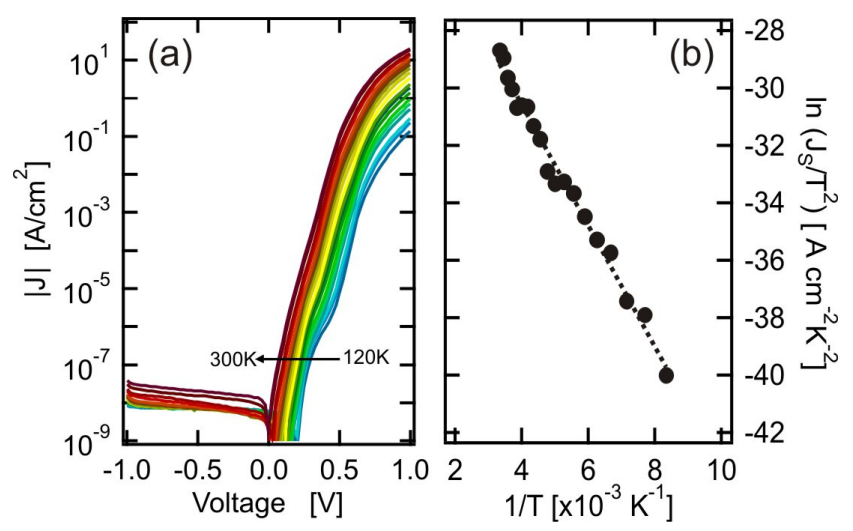

Figure 5: (a) Temperature dependence of the IV characteristics a Pd Schottky diode and (b) corresponding Richardson plot. The diode area is $120 \times 100 \mu \mathrm{m}^{2}$.

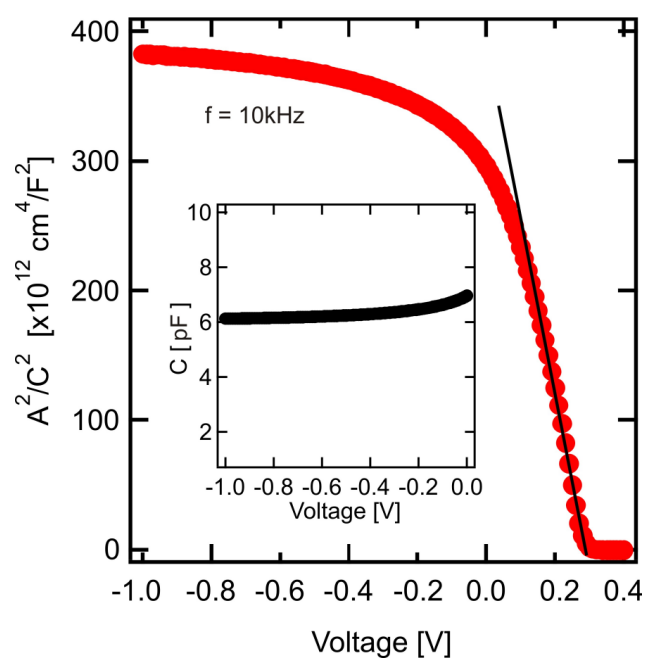

Figure 6: The diodes' CV characteristics. The inset shows the capacitance measured at negative bias: the value is almost contact at $6 \mathrm{pF}$. The diode's area is $120 \times 100 \mu \mathrm{m}^{2}$. 


\begin{tabular}{|c|c|c|}
\hline barrier height & $\mathbf{\Phi}_{\mathbf{B}, \mathbf{I V}}(\mathbf{e V})$ & 0.81 \\
\hline saturation current & $\mathbf{I}_{\mathbf{S}}(\mathbf{p A})$ & 12.2 \\
\hline ideality factor & $\mathbf{n}$ & 1.36 \\
\hline series resistance & $\mathbf{R}_{\mathbf{s}}(\mathbf{\Omega})$ & 238 \\
\hline free charge density & $\mathbf{N}_{\mathbf{e}}\left(\mathbf{c m}{ }^{-3}\right)$ & $5.8 \times 10^{14}$ \\
\hline built-in potential & $\mathbf{\Phi}_{\mathbf{b i}, \mathbf{C V}}(\mathbf{e V})$ & 0.33 \\
\hline barrier height & $\mathbf{\Phi}_{\mathbf{B}, \mathbf{C V}}\left(\mathbf{e V}^{-3}\right)$ & 0.62 \\
\hline $\begin{array}{c}\text { charge density } \\
\text { depletion region }\end{array}$ & $\mathbf{N}_{\mathbf{d e p l}}\left(\mathbf{c m}^{-3}\right)$ & $7.9 \times 10^{15}$ \\
\hline depletion region thickness & $\mathbf{W}(\mathbf{n m})$ & 260 \\
\hline
\end{tabular}

Table I. Characteristics of the Schottky diodes from IV and CV measurements at RT.

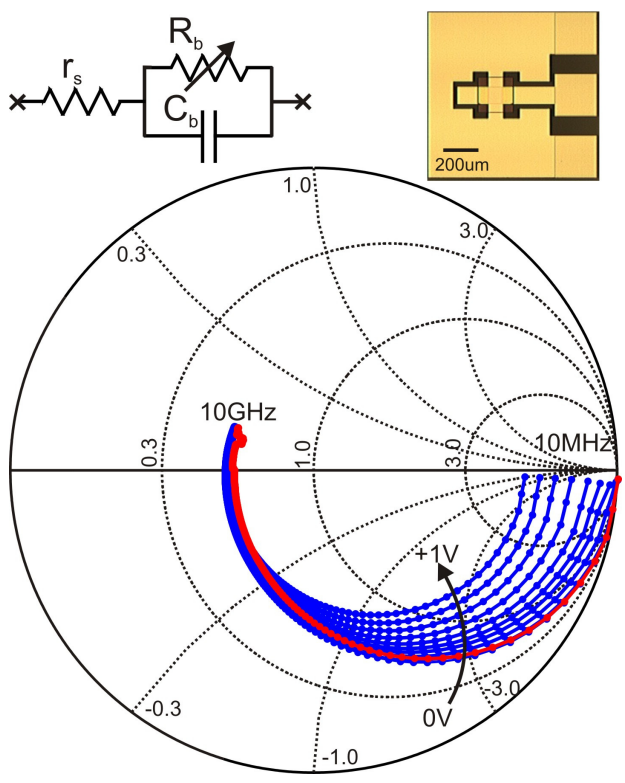

Figure 7: $S_{11}$ parameters from $10 \mathrm{MHz}$ to $10 \mathrm{GHz}$ at different bias voltages. The red curve is the $0 \mathrm{~V}$ measurement. Top-left inset shows the equivalent small signal model of the diode. The top-right image reveals the structure used for these RF measurements.

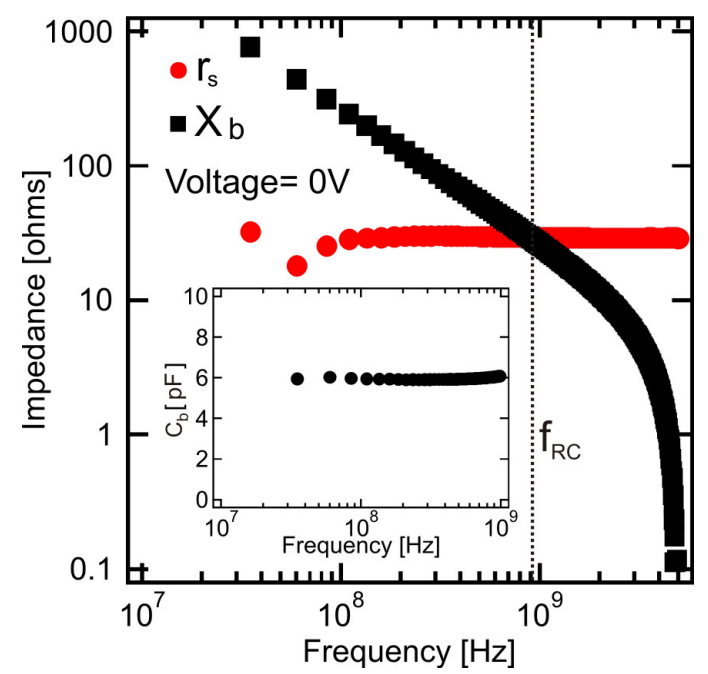

Figure 8: Imaginary and real part of the diode's impedance as a function of the frequency at bias of $0 \mathrm{~V}$. The cut-off frequency $f_{R C}$ of the diode is shown. The inset shows the value of the capacitance $C_{b}$ extracted from $X_{b}$ as a function of the frequency.

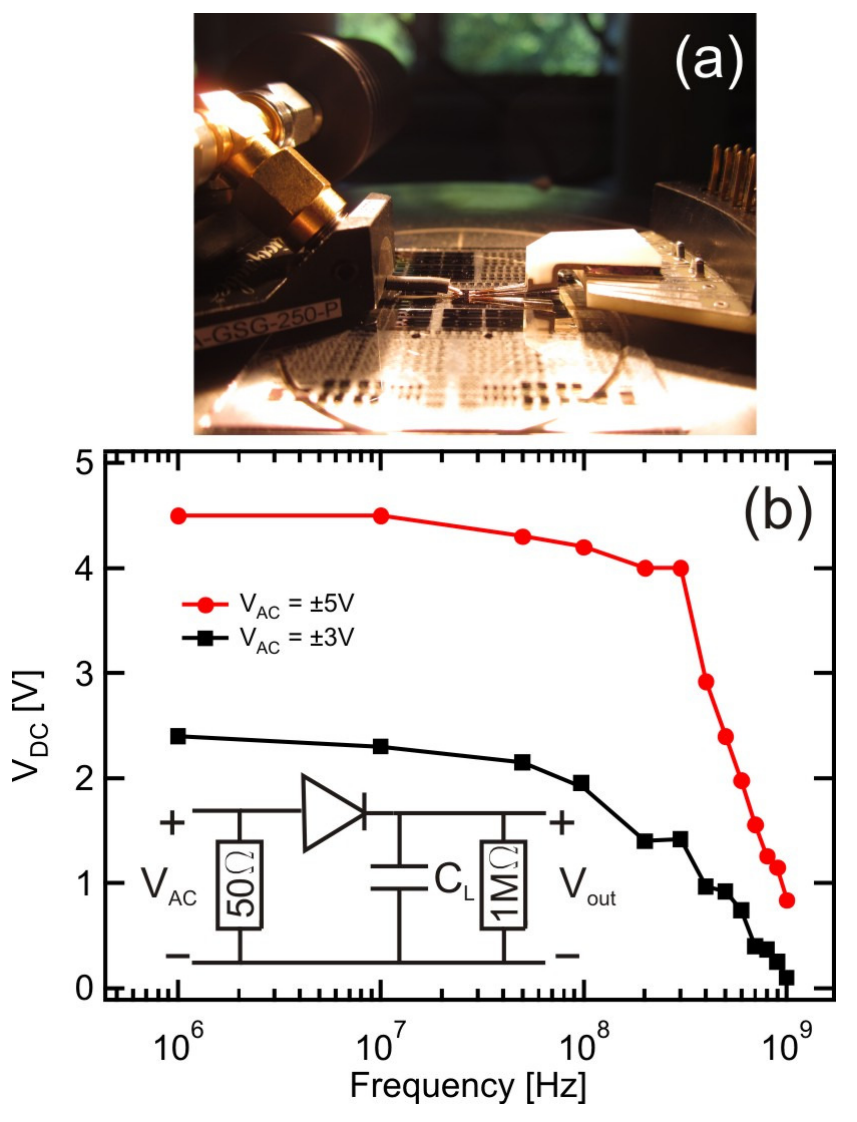

Figure 9: (a) Photograph of the measurement setup and (b) average DC component of the rectified output signal $\left(\mathrm{V}_{\text {out }}\right)$ as a function of the applied AC frequency signal for two different $\mathrm{AC}$ amplitudes. The inset shows the rectifier schematic.
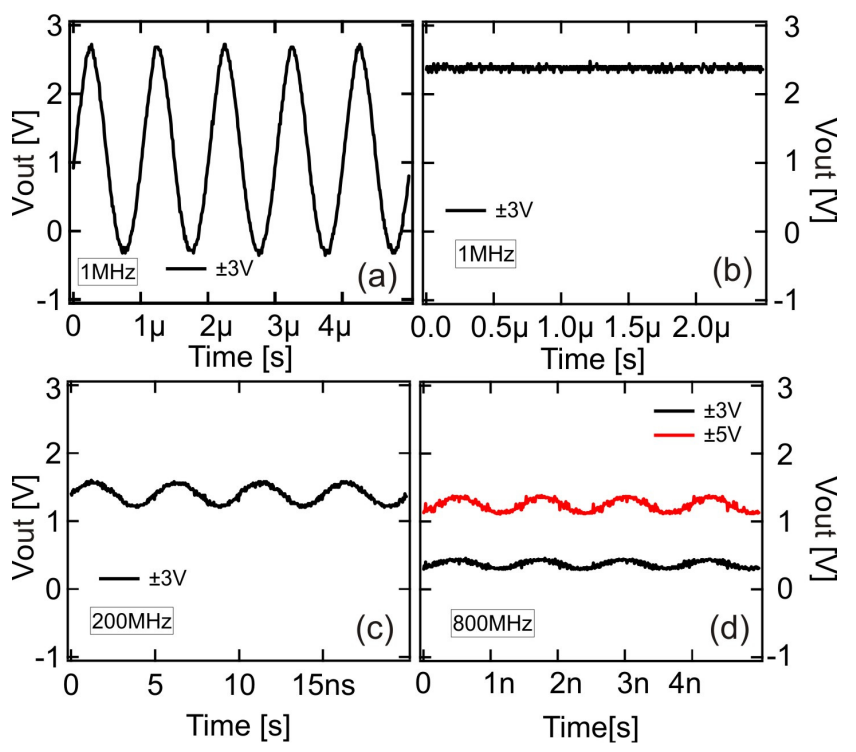

Figure 10: Rectified voltage ( $\mathrm{V}_{\text {out }}$ of the inset of Fig.9) at (a) $1 \mathrm{MHz}$ and $\mathrm{C}_{\mathrm{L}}=0$ (b) $1 \mathrm{MHz}$ and $\mathrm{C}_{\mathrm{L}}=100 \mathrm{nF}$, (c) $200 \mathrm{MHz}$ and $\mathrm{C}_{\mathrm{L}}=100 \mathrm{nF}$, (d) $800 \mathrm{MHz}$ and $\mathrm{C}_{\mathrm{L}}=100 \mathrm{nF}$. 